\title{
MOBILE AGENTS AND SECURITY: PROTOCOLS FOR INTEGRITY
}

\section{Antonio Corradi ${ }^{1}$, Marco Cremonini ${ }^{1}$, Rebecca Montanari ${ }^{1}$ and Cesare Stefanelli ${ }^{2}$}

\author{
${ }^{1}$ Dipartimento di Elettronica, Informatica e Sistemistica - Università di Bologna \\ Viale Risorgimento 2- 40136 Bologna - ITALY \\ ${ }^{2}$ Dipartimento di Ingegneria - Università di Ferrara \\ Via Saragat 1- 44100 Ferrara - ITALY
}

\{acorradi, mcremonini, rmontanari\}@deis.unibo.it, cstefanelli@ing.unife.it

\begin{abstract}
The Mobile Agent paradigm seems to be a promising and innovative technology for developing applications in open, distributed and heterogeneous environments because it can overcome some of the limits of traditional Client/Server approaches. Many application areas, such as e-commerce, mobile computing, network management and information retrieval can benefit from the application of the MA technology. The widespread use of mobile agents is currently mainly limited by the lack of security, a requirement that should be faced when dealing with the Internet untrusted environment. The paper focuses on the problem of ensuring the integrity of agents in these environments and presents a range of solution strategies. In particular, it describes and compares two different approaches to achieve agent integrity. The first one makes use of a Trusted Third Party entity, while the second one is based on a distributed protocol that does not assume any secure collaborating entity. The two solutions suite different areas and we have integrated them in a flexible environment, called Secure and Open Mobile Agent (SOMA), that can support a wide range of applications.
\end{abstract}

Keywords: Mobile agents, Internet, security and integrity, TTP protocols, multiplehops protocol

\section{INTRODUCTION}

The development of applications for open, distributed and heterogeneous systems has motivated the exploration of new execution models based on mobile and dynamic entities [4]. In particular, the Mobile Agent (MA) paradigm has attracted interest, for its flexibility and suitability in dynamic and heterogeneous scenarios. Some intrinsic 
features of MA, such as autonomy, efficiency and easy personalisation, provide new solution strategies for many application areas, i.e., e-commerce, mobile computing, network management and information retrieval, within global and open environments such as the Internet.

However, MA adoption is currently limited by the lack of a thorough security framework. Security is an essential issue in mobile agent systems: both agents and their hosting sites of execution should be protected and secure operations granted.

MA research has mainly focused on how to protect sites from potentially malicious agents [13]. The agents must be prevented from accessing to information they are not authorised to obtain, causing a denial of service to other authorised entities, and interfering with agents of other users. The most common solutions rely on the cryptographic authentication and authorisation mechanisms usually employed in distributed systems [10].

The complementary issue of protecting agents, in terms of integrity and privacy, from malicious execution environments has not received the same attention. Its complexity makes difficult the development of comprehensive technical solutions [8], [13] [14]. Agent protection is, however, a crucial concern: without an appropriate security level, MA applications could only execute in trusted environments, and could not be considered in global and open scenarios.

This paper focuses on the agent integrity issue. It proposes two solutions for ensuring integrity to agents traversing several sites of execution before going back to their originating sites. The first solution requires the presence of a Trusted-ThirdParty (TTP) entity offering a trusted and secure environment to agents in need of performing cryptographic functions [10]. In this case, after any visit to any untrusted site, an agent should visit a TTP site to validate its computation. The second solution, called Multiple-Hops (MH), achieves agent integrity without the need of a TTP. The $\mathrm{MH}$ protocol does not impose additional hops to agent paths: agents are able to autonomously roam throughout the network without being constrained to interact with TTPs. The two solutions are based on different assumptions and are suitable for different application areas. Their integration can provide an efficient tool for a wide range of application areas.

The paper shows how the TTP solution and the MH protocol have been implemented in our Secure and Open Mobile Agent (SOMA) environment (available from http://www-lia.deis.unibo.it/Software/MA/), that has been designed and implemented by considering security as a key property to be accounted for and integrated at any system layer [2]. A performance analysis is also presented.

\section{SECURITY ISSUES IN MOBILE AGENT APPLICATIONS}

The security infrastructure should protect both sites against malicious behaviour of agents and agents against potentially malicious sites. Many proposals address the issue of site protection against malicious agents. In the Java area, the sandbox model and its evolution aim to confine the scope of executable content [5]. Other approaches exploit type safe languages [13] or associate mobile code with the proof of its behaviour allowing sites to verify its correctness before executing it [11].

With regard to the complementary problem of protecting agents executing in untrusted and possibly malicious sites, there are no accepted and standardised 
solutions currently available and only a few proposals. The main issues to be comprehensively addressed are agent integrity and privacy.

Agent integrity requires the identification of agent tampering, either in code or in state parts, by malicious sites. Currently, the research in this area proposes approaches for both prevention and detection of tampering. With regard to the prevention of agent state tampering, few proposals mainly rely on special tamperproof hardware, with the drawbacks of clashing with openness and limited scalability of applications [14]. Among the proposals that adopt a strategy of a-posteriori detection of tampering, some employ centralised solutions which rely on TTP entities. The TTP can be used to track the agent execution at each intermediate step by recording partial results [13]. Others use a cooperating agent as a TTP executing on sites identified as trusted, with the goal to securely record agent itinerary [12]. The central role of TTPs may cause some inefficiencies that can be overcome by more distributed protocols. According to this consideration, some solutions partially achieve integrity by replicating agents and by exploiting replication to compare the results obtained by following different paths [15]. Other solutions present cryptographic approaches to make possible to the agent owner to ascertain whether all intermediate sites have behaved correctly [8].

With regard to agent privacy, of both the code and the state parts, it seems extremely difficult to guarantee it. The agent code cannot be hidden from the site responsible for its execution. The same applies to the data part if the site has to work on it, even if now some researches address these issues [9], [13].

\section{THE AGENT INTEGRITY PROBLEM}

The basic property of any protocol for agent integrity is to ensure that no attack aimed at modifying or deleting the agent code and state goes undetected. We distinguish between integrity mechanisms for agent code and for agent state. Code integrity can be achieved with traditional cryptographic methods with the assumption of code invariance. State integrity is harder to ensure, because the state is likely to change during agent itinerary in order to contain the data collected at each visited site.

This section focuses on the problem of achieving agent state integrity, by presenting and comparing two solutions. The first one represents a centralised approach that relies on TTP entities that have to validate the new agent state at each hop. The second solution is based on the distributed $\mathrm{MH}$ protocol that avoids single point of control. The two approaches present the following common assumptions: $(i)$ agents can dynamically decide the sites to visit and are not constrained to follow only paths predefined by their owners; (ii) only a certain percentage of sites visited by an agent might be malicious; this assumption is generally considered reasonable and realistic [12]; (iii) there is a public-key infrastructure, managing entity certification [10]; (iv) any couple of sites can be connected via an encrypted channel to preserve state confidentiality.

Furthermore, the two solutions share the following definitions: (i) a Message Integrity Code (MIC) permits to verify agent state integrity; a MIC is a hash over some data with a collision free hash function; (ii) an agent is composed by three parts: Code and 
Initialisation Data (CID), Application Data (AD), and Protocol Data (PD). The CID is the immutable part of the agent. The $\mathrm{AD}$ part contains the data collected by the agent at each hop. The integrity of collected data is ensured by the PD part that records the information needed for the verification of integrity.

\subsection{The TTP solution to agent integrity}

We have first experienced a protocol based on the presence of Trusted Third Parties. In our SOMA framework TTP functionality are embodied in a specific mobile agent residing on a trusted site. This agent collaborates with application agents that roam through remote untrusted sites.

Figure 1 shows the TTP protocol for agent integrity and the principals involved: we distinguish the sender site (Sender), the Trusted Third-Party (TTP), and the untrusted remote sites $S_{i}(i=1, \ldots, N)$. In the TTP protocol, before moving to an untrusted site, the agent has to visit the TTP, in charge of compute and record a MIC of the data that the agent previously collected and stored in the PD part. Once the agent arrives on the untrusted site, new data can only be stored in the agent $\mathrm{AD}$ part. Then, the agent goes back to the TTP, where a new integrity-protected state of the agent is obtained by adding new $\mathrm{AD}$ values to the previously collected one. Finally, the TTP computes and records a new MIC on the PD part. The detailed steps are shown in the following.

Initial Step: The Sender computes the first digest $\mathrm{MIC}_{1}$ of the agent (AppAg). Then the agent is sent to the first remote host $S_{1}$.

$$
\begin{aligned}
& \mathrm{AD}_{1}=\text { void } ; \mathrm{PD}_{1}=\mathrm{MIC}_{1} \\
& \text { Sender } \rightarrow \mathrm{S}_{1}: \mathrm{AppAg}=\left(\mathrm{CID}, \mathrm{PD}_{1} A D_{1}\right)
\end{aligned}
$$

From the $S_{i}$ untrusted site to the TTP. The agent collects new data $f\left(S_{i}\right)$ from the untrusted site and stores it in the $\mathrm{AD}$ part before migrating to the TTP.

$$
\begin{aligned}
& \mathrm{AD}_{i}=\mathrm{f}\left(\mathrm{S}_{i}\right) \\
& \mathrm{S}_{i} \rightarrow \mathrm{TTP}: \text { AppAg }=\left(\mathrm{CID}, \mathrm{PD}_{i}, A D_{i}\right)
\end{aligned}
$$

At the TTP. The TTP verifies the digest $\mathrm{MIC}_{\mathrm{i}}$ (computed and recorded by the TTP in the last agent migration) checking for any malicious modification to the PD data previously stored by the agent. This is achieved by calculating a fresh MIC on the agent $\mathrm{PD}$ part, and comparing it with the one previously recorded. Then, the PD part $\left(P D_{i+1}\right)$ can be enlarged with the new data $P D_{i}+A D_{i}$ collected in the untrusted site. $A$ new digest $\mathrm{MIC}_{\mathrm{i}+1}$ is computed and recorded on both the TTP and the agent.

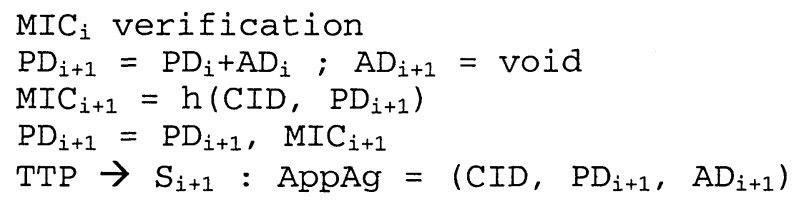

The steps are repeated for any untrusted party, up to return to the original Sender with all the gathered data. 


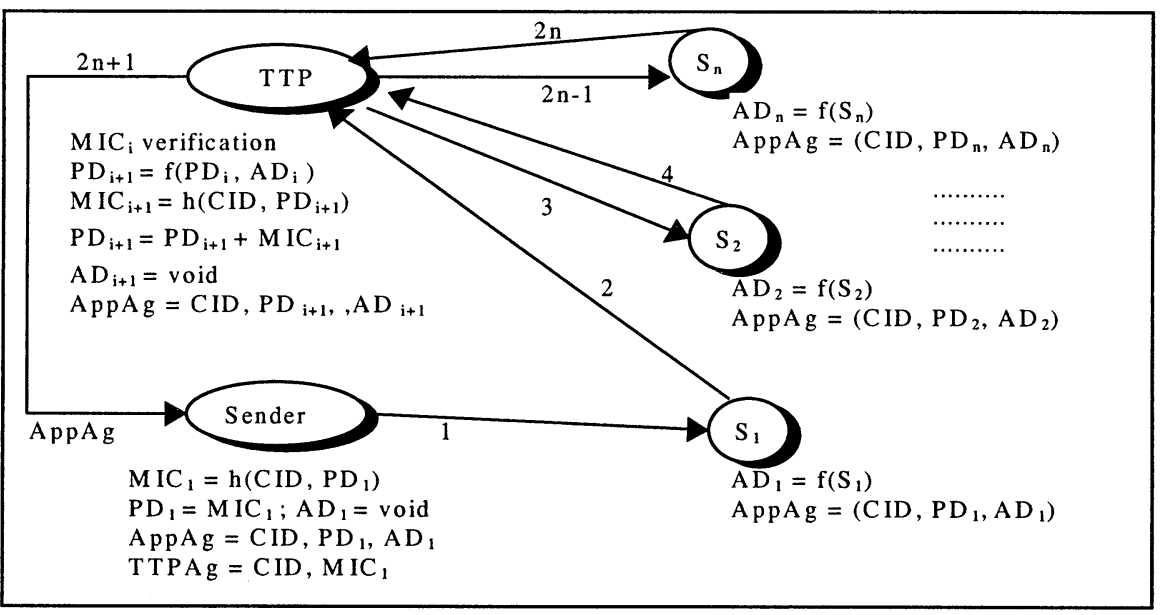

Figure 1. The TTP solution for mobile agent integrity.

It is easy to see that the presence of the TTP agents makes possible to achieve the integrity of the agents. The information collected by the agent can not be modified or deleted because each cryptographic function is delegated to the TTP and also the destruction of the agent could be tracked querying the TTP. Moreover, the collusion of two malicious hosts is ineffective, having only the possibility to modify the AD part without violating the integrity check.

In many situations an application may require to obtain results from the agents even in presence of a protocol failure due, for instance, to a malicious interference. This could be achieved only with protocols, as the TTP one, that do not introduce dependencies in agent state between subsequent hops. TTP provides intermediate results, which cannot be affected by a successive protocol failure. We also point out that the agent is not forced to always deal with the same TTP but, by means of the embodied MIC, is free to exploit different TTPs.

It is worth to notice that the above protocol has some drawbacks. In fact, it imposes a high overhead, by requiring the agent to move to the TTP after each visit of an untrusted site. In addition, the TTP protocol scalability is undoubtedly limited by the availability of trusted nodes, which implement the TTP side of the protocol. Generally, it is reasonable to assume the availability of TTPs when agents roam through sites with reciprocal cooperating relations, for instance sites of one organisation. Otherwise, taking into account an open and global Internet space, the TTP approach becomes less appropriate.

\subsection{The Multiple-Hops protocol for agent integrity}

We have designed an alternate fully distributed solution, called Multiple-Hops $(\mathrm{MH})$ protocol, that does not require any TTP. The mobile agent is free to migrate among several sites without being constrained at each hop to interact with a TTP. At each 
site, the agent collects a partial data and appends it to the previous ones in its $\mathrm{AD}$ part. Each site, on its turn, must provide a cryptographic proof of the agent computation, which is stored in the agent PD part. A cryptographic proof chain is, thus, produced at the end of agent itinerary and is verified by the Sender when the agent moves back to it to ensure agent state integrity. The protocol steps are as follows (see Figure 2).

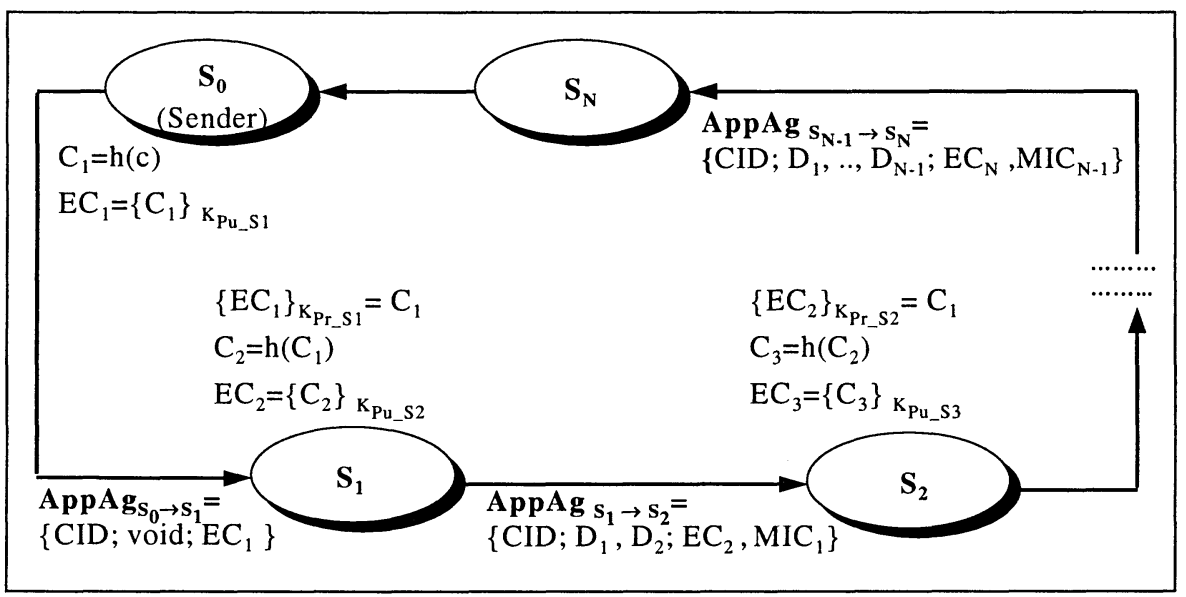

Figure 2. The $\mathrm{MH}$ protocol for mobile agent integrity.

Initial Step. The Sender computes $\mathrm{C}_{1}=\mathrm{h}(\mathrm{C})$, where $\mathrm{c}$ is a random number and $\mathrm{h}$ a collision free hash function and sends it to the first site encrypted with the public key of the site $\left(\mathrm{EC}_{1}\right) . \mathrm{C}_{1}$ acts as a secret shared between the first destination place and the Sender.

$$
\begin{aligned}
& \mathrm{C}_{1}=\mathrm{h}(\mathrm{C}) ; \mathrm{EC}_{1}=\text { encrypted } \mathrm{C}_{1} \\
& \mathrm{AD}_{1}=\text { void } ; \mathrm{PD}_{1}=E C_{1} \\
& \text { Sender } \rightarrow \mathrm{S}_{1}: \mathrm{AppAg}=\left(\mathrm{CID} ; \mathrm{AD}_{1} ; \mathrm{PD}_{1}\right)
\end{aligned}
$$

From $S_{i}$ to site $S_{i+1}$. Any intermediate site $S_{\mathrm{i}}, \mathrm{i}=\{1 \ldots \mathrm{N}\}$, that hosts the mobile agent execution, decrypts the received secret $C_{i}$. The agent appends the data locally collected $\left(D_{i}\right)$ to the $A D$ part.

To ensure data integrity $S_{i}$ computes $\mathrm{MIC}_{i}=h\left(C_{i}, D_{i}, \mathrm{MIC}_{i-1}\right)$, then, it calculates $C_{i+1}=h\left(C_{i}\right)$ for the next place $S_{i+1}$ while the old $C_{i}$ is deleted. The $\mathrm{MIC}_{i}$ and the encrypted $\mathrm{C}_{i+1}$ are encapsulated in the PD part of the agent. This step is repeated for any intermediate site.

$$
\begin{aligned}
& C_{i+1}=h\left(C_{i}\right) ; E C_{i+1}=\text { encrypted } C_{i+1} \\
& M I C_{i}=h\left(C_{i}, D_{i}, M I C_{i-1}\right) \\
& \mathrm{AD}_{i}=D_{i} ; \mathrm{PD}_{i}=E C_{i+1}, M C_{i} \\
& \mathrm{~S}_{i} \rightarrow \mathrm{S}_{i+1}: \text { AppAg }=\left(C I D ; A D_{i} ; \mathrm{PD}_{i}\right)
\end{aligned}
$$


The Sender. As the last hop, the agent goes back to the Sender that is in charge of verifying the integrity of the MIC encapsulated chain. The verification is performed by calculating a fresh $\mathrm{MIC}_{\mathrm{N}}$. The initials random number and all the data collected are used to compute it. If the fresh $\mathrm{MIC}_{N}$ does not match with the received one, some partial information have been deleted or modified by some intermediate malicious sites.

It is straightforward to see that $\mathrm{MH}$ ensures forward agent integrity [15]: it is not possible for the malicious site $S_{i}$ to modify or delete $D_{k}$, with $1<k<i$, without being detected by the Sender. The lack of knowledge of the previous secrets $C_{k}$, with $1<k$ $<\mathrm{i}$, prevents the place $S_{\mathrm{i}}$ from being able to tamper previously collected data; in fact, each hashed secret is known only to the corresponding intermediate site and the Sender. In this way, any site can not forge previous MICs as it cannot rebuild the correct sequence of cryptographic proofs due to its partial knowledge. In addition, given a certain chain of collected data $D_{k} \ldots . . D_{k+n}$, it is not possible for an attacker to arbitrarily insert its own data between $D_{k} \ldots D_{k+n}$; any arbitrary insertion invalidates, in fact, the whole data chain.

The $\mathrm{MH}$ protocol answers to many mobile agent application requirements, such as autonomy and efficiency, by not constraining the agent to interact with a TTP entity.

The protocol has some drawbacks, though. In case of protocol failure, all partial results, related to a subset of the remote visited nodes, are completely lost. Furthermore, the $\mathrm{MH}$ protocol works properly only with the 'visit-once' assumption, i.e., each intermediate site hosts the agent no more than once. If an agent visited the same site twice, the site could have saved the first received secret and could abuse of this to partially or totally replace the data chain with a fake one or to delete it without being detected by the Sender. Similar considerations apply in the case of collusion between multiple sites.

However, we claim that the 'visit-once' assumption is suitable for those application areas where there is no need to re-contact previously visited sites (information retrieval, comparison shopping), whereas it excludes applications based on auctions and negotiations. Moreover, we consider the assumption of absence of collusion between sites reasonable in a competitive scenario.

\subsection{Comparison and Integration of TTP and MH Protocols}

The TTP solution provides a more fault tolerant solution than the MH protocol, because does not introduce dependencies between hops: in case of problems, either fault or malicious interference, the intermediate results can be made available to the application. However, the TTP relies on the presence of trusted sites throughout the domain where the agents roam. When trusted sites are not available, agent integrity can be provided by the $\mathrm{MH}$ protocol.

There are cases where neither the TTP nor the MH approach offers the best solution in term of efficiency. A combined approach allows to overcome the main drawbacks of the two protocols by integrating TTP with MH.

In this combined approach the agent itinerary is partitioned into smaller partial paths. Within one partial path, the agent integrity is achieved with the MH protocol. When the mobile agent visits the last site in the partial path, it is forced to migrate to 
the TTP. After the integrity of collected data is verified, the TTP generates a new secret to be used in the subsequent path, encrypts it with the public key of the subsequent site and calculates a new integrity-protected state of the agent. The description of the combined approach steps follows (the comments refer to the modifications requested by the integration):

Initial Step

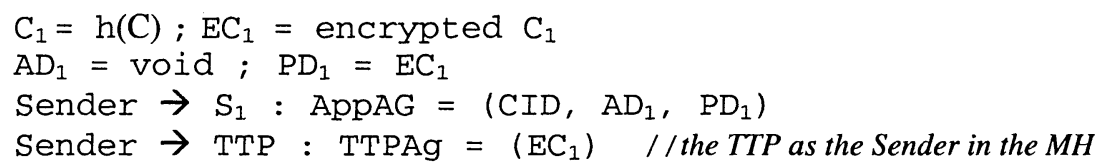

Protocol MH Steps : from $S_{i}$ to site $S_{i+1}$ or to the TTP

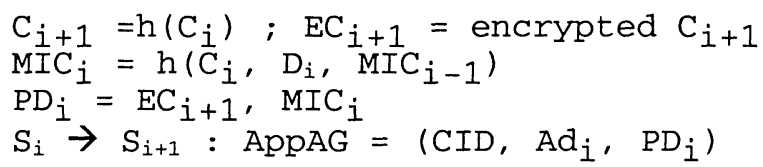

Protocol TTP Steps : at the TTP

$M I C_{j}$ verification // verification of the chained $M I C_{j}$

$C_{j+1}=h\left(C_{\text {TTP }}\right) \quad / /$ a new secret for the subsequent path is generated

$\mathrm{EC}_{j+1}=$ encrypted $\mathrm{C}_{j+1}$

$A D_{j+1}=A D_{j}$

$\mathrm{MIC}_{j+1}=h\left(C_{j+1}, A D_{j+1}\right)$

Protocol TTP Steps : from the TTP to the $S_{j+1}$ untrusted site

$\mathrm{PD}_{j+1}=\mathrm{EC}_{j+1}, \mathrm{MIC}_{j+1}$

$\mathrm{TTP} \rightarrow \mathrm{S}_{j+1}: \mathrm{AppAG}=\left(\mathrm{CID}, \mathrm{AD}_{j+1}, \mathrm{PD}_{j+1}\right)$

In this solution the degradation of performance that a pure TTP approach introduces is considerably reduced because the TTP is involved only in a limited number of hops. The combined approach allows to extract partial results even in case of protocol failure. By exploiting TTP, in fact, the MH protocol robustness is improved: the chain of collected data is partitioned in intermediate checkpoints (all the TTP steps) recording the partial state of the application. Finally, the 'visit-once' assumption of $\mathrm{MH}$ could be relaxed with the introduction of TTP. Even cycles in agent itineraries could be prevented by forcing the agents to migrate to a TTP before revisiting a site. The TTP, by generating a new initial encrypted secret carried by the agent, prevents the data chain from being replaced by a fake one.

The combined solution enlarges the flexibility of application design by making possible to achieve agent integrity in a wider application scenario.

\section{THE SECURE AND OPEN MOBILE AGENT ENVIRONMENT}

The TTP and Multiple-Hops protocols for agent integrity have been integrated in SOMA, a secure environment for mobile agent programming.

SOMA answers to the requirements of a wide range of open, untrusted, and global distributed systems, ranging from simple LANs to complex architectures variously interconnected by bridges, routers, gateways, and firewalls. The SOMA support 
provides a hierarchy of abstraction localities suitable for describing any kind of internetwork scenario. SOMA abstraction localities represent physical resources: agents execute in places that represent physical nodes; any physical node hosts at least one place for agent execution; places are grouped in domain abstractions that can represent a LAN, and can be interconnected by using gateways [1] [2].

With regard to agent coordination, agents inside a place can interact by sharing common resources. Whenever one agent needs to share one resource with another agent residing in a remote place, it is forced to migrate to that remote place. Outside the scope of the place, agents can interact only via message passing. The SOMA support guarantees that messages are delivered to agents even in case of migration.

\subsection{SOMA security}

Security is a primary concern of SOMA that considers both the protection of sites against malicious agents and the protection of agents against malicious sites.

With regard to place protection, the definition of different locality abstractions permits to enforce layered security policies. At the domain level, there is a global security policy, which imposes general authorisation rules; each place can only apply restrictions to the security policy of the domain of belonging. Security policies state the permissions assigned to agents depending on their credentials. When entering a place, agents are authenticated and authorised to operate on node resources only by invoking the operations provided by their interfaces.

With regard to agent protection, SOMA protects agents when in transit over insecure channels by using encrypted and authenticated channels [4]. The SOMA project faces the agent integrity protection by implementing the TTP and MH integrity protocols.

As a final consideration, let us note that security is not without cost. SOMA presents a wide variety of available security mechanisms and gives the possibility to decide a suitable trade-off between security needs and required performance.

\subsection{SOMA Implementation}

SOMA is implemented in Java to exploit its easy integration with the Web scenario, its intrinsic portability and interoperability in heterogeneous environments: we have developed the system on SUN workstations, and easily ported it to PCs. Our support uses the JDK 1.2 beta2 [7].

With regard to SOMA architecture, the object-oriented nature of Java has helped in the design: the encapsulation concept suits the locality abstraction needs of both resources and agents; the classification principle makes possible to inherit behaviour from already specified components. A well-known problem of Java is the lack of full mobility support, especially for Java threads: it is not possible to save the whole state of a thread before its migration to a different node. This restriction has been overcome by providing a go operation at the application level that allows one agent to move itself during its execution, by specifying the method to be activated after the migration. The go operation can embed also the $\mathrm{MH}$ protocol steps. 
SOMA exploits the security mechanisms available in JDK 1.2 and the cryptography extensions in the IAIK-JCE 2.0 package to provide the needed degree of protection [6].

Java provides the basic mechanisms and internal strategies to design a specific model of trust to protect sites against agents [5]. The authentication of mobile agents uses the DSA algorithm with X.509 certificates. The authorisation is based on ACL mechanisms. The integrity check can employ either MD5 or SHA1 or the functions enclosed in IAIK-JCE 2.0. The confidentiality of agent information, when needed, is granted by encrypting/decrypting any agent with DES and SSL.

\section{PROTOCOL PERFORMANCE}

The TTP and the MH solutions ensure agent integrity by incurring in execution and transmission overheads. While the execution cost is due to cryptographic operations, the transmission cost considers the increased network bandwidth overhead and latency with regard to the case of agents without any security mechanisms.

We first compare the protocols by considering the execution cost of one agent that gathers partial application data $(\mathrm{AD})$ of equal size in each visited site.

In the TTP solution, the cryptographic functionality are performed by the TTP, that experiences the execution overhead of $2 \mathrm{~T}_{\mathrm{MIC}}$, needed to verify the agent MIC and to compute a new one. Hence, in case of $\mathrm{N}$ visited sites, the cost is:

$\mathrm{T}_{\text {TOTEXEC-TTP }}=\mathrm{N} 2 \mathrm{~T}_{\text {MIC }}$

In the $\mathrm{MH}$ protocol, the total overhead depends on two terms: (i) the cost due to the intermediate sites ( $\mathrm{T}_{\text {TOTHOPS-MH }}$ ) and (ii) the overhead introduced by the Sender to verify the agent integrity $\left(\mathrm{T}_{\text {SENDER-MH }}\right)$.

Considering the first term, the overhead introduced by each intermediate site can be expressed as: $T_{\text {HASH }}+T_{\text {MIC }}+T_{\text {DECRYPT }}+T_{\text {CRYPT }}$. Where $T_{\text {HASH }}$ indicates the cost of making the hash of the received secret, $\mathrm{T}_{\mathrm{MIC}}$ is the cost of computing the MIC and $\mathrm{T}_{\mathrm{DECRYPT}} / \mathrm{T}_{\mathrm{CRYPT}}$ are the costs of decrypting/encrypting the secret for the next site. Considering the above terms, we can observe that they depend on the correspondent input data size. $T_{M I C}$ is the most significant part, depending mainly on the intermediate application data $(\mathrm{AD})$. $\mathrm{T}_{\mathrm{HASH}}, \mathrm{T}_{\mathrm{DECRYPT}}$ and $\mathrm{T}_{\mathrm{CRYPT}}$ depend on the secret size which is fixed and negligible if compared with $\mathrm{AD}$. Thus, in the case of $\mathrm{N}$ visited sites, the $\mathrm{MH}$ protocol requires a total execution overhead of:

$$
\mathrm{T}_{\text {TOTHOPS-MH }}=\mathrm{N} \mathrm{T}_{\text {INT-MH }}=\mathrm{N}\left(\mathrm{T}_{\text {HASH }}+\mathrm{T}_{\text {MIC }}+\mathrm{T}_{\text {DECRYPT }}+\mathrm{T}_{\text {CRYPT }}\right) \cong \mathrm{NT}_{\text {MIC }}
$$

The Sender, when finally the agent returns back, has to verify the integrity of the encapsulated MIC chain, which requires:

$$
\mathrm{T}_{\text {SENDER-MH }}=\mathrm{N}\left(\mathrm{T}_{\mathrm{HASH}}+\mathrm{T}_{\mathrm{MIC}}\right) \cong \mathrm{NT}_{\mathrm{MIC}}
$$

where $T_{\text {HASH }}$ is the cost to hash any successive secret and $T_{\text {MIC }}$ is the cost to calculate the MIC of any partial application data received back. As in the previous case, $\mathrm{T}_{\mathrm{MIC}}$, depending on the application data size, is the dominant term. 


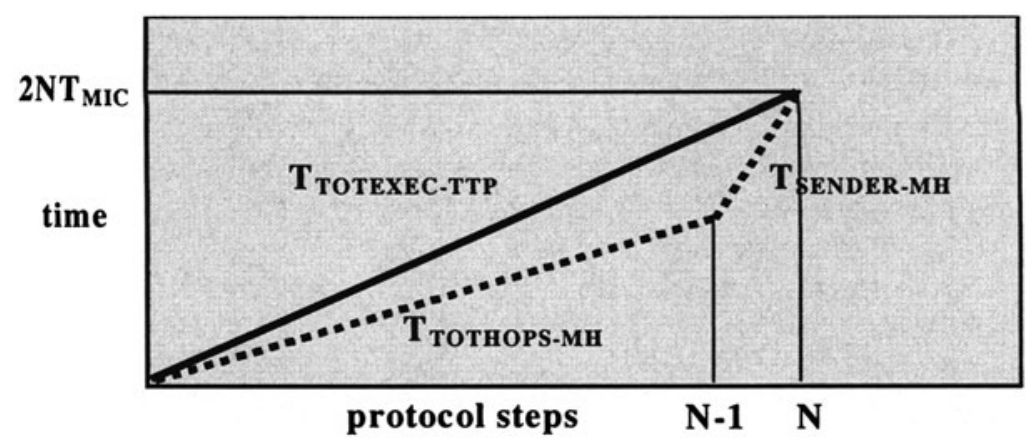

Figure 3. Execution cost distribution (in $\mathrm{msec}$ ).

The above results show that while the execution overheads imposed by TTP and $\mathrm{MH}$ protocols are similar (both are approximately $2 \mathrm{NT}_{\mathrm{MIC}}$ ), they grow differently (see Figure 3). In fact, while the TTP solution distributes the cost uniformly by performing an integrity verification at each hop, the $\mathrm{MH}$ protocol presents a nonuniform cost distribution by verifying the agent integrity only at the Sender site.

We now consider the transmission cost. We assume that the transmission cost grows linearly with the agent size. Thus, the transmission cost between two sites of an agent without integrity protection (with its $\mathrm{CID}, \mathrm{PD}$ and $\mathrm{AD}$ parts), can be expressed as:

$$
\mathrm{T}_{\mathrm{TX}}=\mathrm{T}_{\mathrm{CIDTX}}+\mathrm{T}_{\mathrm{ADTX}}+\mathrm{T}_{\mathrm{PDTX}}=\alpha \mathrm{D}_{\mathrm{CID}}+\beta \mathrm{D}_{\mathrm{AD}}+\gamma \mathrm{D}_{\mathrm{PD}}
$$

where $D_{C I D}, D_{A D}$ and $D_{P D}$ are the size of agent parts and $\alpha, \beta, \gamma$ are constant values.

The total transmission cost $\mathrm{T}_{\text {TOTTX }}$ of each integrity protocol in a visit of $\mathrm{N}$ untrusted sites is obtained by considering the enlarged agent size strictly due to the protocol. We also consider the agent size increasing due to the data collected in remote sites.

MH Protocol Transmission Cost. In the $\mathrm{MH}$ protocol, the agent size increases at each step because of the new collected data $\mathrm{D}_{\mathrm{AD}}$ and the MIC stored in PD. The latter term introduces a negligible cost if compared with $\triangle \mathrm{D}_{\mathrm{AD}}$.

The total transmission cost of the protocol $\mathrm{MH}$ for $\mathrm{N}$ untrusted visited sites could be expressed as:

$$
\begin{gathered}
\mathrm{T}_{\mathrm{TOTTX}-\mathrm{MH}}=\mathrm{N} \alpha \mathrm{D}_{\mathrm{CID}}+\mathrm{N} \beta \mathrm{D}_{\mathrm{PD}}+\gamma \Sigma_{0}{ }^{\mathrm{N}} \Delta \mathrm{D}_{\mathrm{AD}}=\mathrm{N} \alpha \mathrm{D}_{\mathrm{CID}}+\mathrm{N} \beta \mathrm{D}_{\mathrm{PD}}+\gamma\left(\Delta \mathrm{D}_{\mathrm{AD}}+\right. \\
\left.\left.2 \Delta \mathrm{D}_{\mathrm{AD}}+\ldots+(\mathrm{N}-1) \Delta \mathrm{D}_{\mathrm{AD}}+\mathrm{N} \Delta \mathrm{D}_{\mathrm{AD}}\right)=\mathrm{N} \alpha \mathrm{D}_{\mathrm{CID}}+\mathrm{N} \beta \mathrm{D}_{\mathrm{PD}}+\gamma \mathrm{N}(\mathrm{N}+1) / 2\right) \Delta \mathrm{D}_{\mathrm{AD}}
\end{gathered}
$$

The last term is dominant in the cost evaluation, then:

$$
\mathrm{T}_{\mathrm{TOTTX}-\mathrm{MH}} \cong \gamma(\mathrm{N}(\mathrm{N}+1) / 2) \Delta \mathrm{D}_{\mathrm{AD}}
$$

TTP Protocol Transmission Cost. The TTP total transmission cost is increased by the additional steps to go back to the TTP between any visit to untrusted sites. In particular, with $\mathrm{N}$ untrusted sites to visit, the agent has to migrate $\mathrm{N}-1$ times back to 
the TTP. Hence, the total number of steps is $2 \mathrm{~N}-1$. The transmission cost can be expressed:

$$
\mathrm{T}_{\text {TOTTX-TTP }}=(2 \mathrm{~N}-1) \alpha \mathrm{D}_{\mathrm{CID}}+\beta \sum_{0}^{2 \mathrm{~N}-1} \mathrm{D}_{\mathrm{PD}}+\gamma \sum_{0}{ }^{2 \mathrm{~N}-1} \mathrm{D}_{\mathrm{AD}}
$$

When the agent migrates from the TTP to the generic $S_{i}$ untrusted host, the PD part is enlarged of $\triangle D_{A D}$. The $A D$ part increases of $\Delta D_{A D}$ in any hop from $S_{i}$ to the TTP and is cleared when the agent migrates from TTP. The last two terms of $\mathrm{T}_{\text {TOTTX-TTP }}$ are dominant:

$$
\begin{aligned}
& \sum_{0}^{2 \mathrm{~N}-1} \mathrm{D}_{\mathrm{PD}}=0+0+\Delta \mathrm{D}_{\mathrm{AD}}+\Delta \mathrm{D}_{\mathrm{AD}}+2 \Delta \mathrm{D}_{\mathrm{AD}}+2 \Delta \mathrm{D}_{\mathrm{AD}}+3 \Delta \mathrm{D}_{\mathrm{AD}}+3 \Delta \mathrm{D}_{\mathrm{AD}}+\ldots+ \\
& (\mathrm{N}-1) \Delta \mathrm{D}_{\mathrm{AD}}+(\mathrm{N}-1) \Delta \mathrm{D}_{\mathrm{AD}}=\mathrm{N}(\mathrm{N}+1) / 2 \Delta \mathrm{D}_{\mathrm{AD}}
\end{aligned}
$$

The same applies to the term $\Sigma_{0}^{2 N-1} D_{A D}$. With the assumption of $\beta=\gamma, T_{\text {TOTTX-TTP }}$ can be approximated to:

$$
\mathrm{T}_{\mathrm{TOTTX}-\mathrm{TTP}} \cong \gamma \mathrm{N}(\mathrm{N}+1) / 2 \Delta \mathrm{D}_{\mathrm{AD}}+\gamma \mathrm{N}(\mathrm{N}-1) / 2 \Delta \mathrm{D}_{\mathrm{AD}}=\gamma \mathrm{N}^{2} \Delta \mathrm{D}_{\mathrm{AD}}
$$

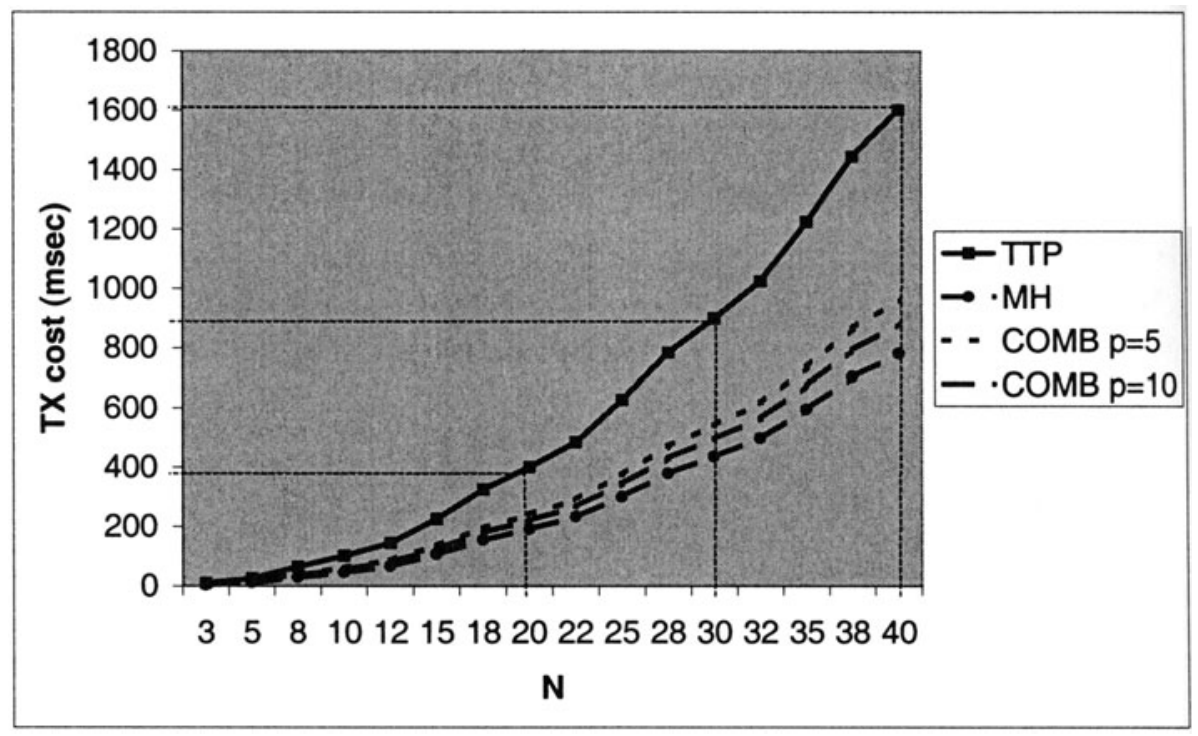

Figure 4. Transmission cost for different number of visited sites.

Combined approach TTP/MH Transmission Cost. In the combined approach, the $\mathrm{MH}$ protocol is used for $p$ hops before a migration to the TTP. In this case, the agent visits $p$ untrusted sites, goes back to the TTP and moves to the new untrusted site. The transmission cost $\mathrm{T}_{\text {TOTтX-сомB }}$ can be expressed in terms of $p$ and of the number of hops to the TTP $\left(\mathrm{N}_{\mathrm{TTP}}=\mathrm{N} / \mathrm{p}-1\right)$ :

$$
\left.\left.\mathrm{T}_{\text {TOTTX-COMB }}=\gamma \mathrm{N}(\mathrm{N}+1) / 2\right) \Delta \mathrm{D}_{\mathrm{AD}}+\gamma \mathrm{p}\left(\mathrm{N}_{\mathrm{TTP}}\left(\mathrm{N}_{\mathrm{TTP}}+1\right) / 2\right)\right) \Delta \mathrm{D}_{\mathrm{AD}}=\gamma \mathrm{N}^{2} / 2(1+1 / \mathrm{p})
$$


Figure 4 shows a comparison of the transmission costs of TTP, MH and the combined approach, considering different values of $N, \Delta D_{A D}=20 \mathrm{~K}$ and $\gamma=1$.

\section{CONCLUSIONS}

Autonomy, efficiency and easy personalisation are distinguished properties of the MA paradigm that can help in information gathering, filtering, brokering and network management. However, despite the advantages offered by MA systems, a wider diffusion of their general use is currently limited mainly by the lack of a comprehensive security framework suitable to address the protection of both agents and sites of execution. One of the most difficult problem to solve is the protection of agent integrity. We propose two solutions for detecting any agent integrity violation, the Trusted Third Party and the Multiple-Hops protocol. The TTP protocol achieves its goal by exploiting a dedicated agent on a trusted site and does not force the roaming agent to depend on the correctness of all the interaction with untrusted sites. Differently, the $\mathrm{MH}$ protocol ensures mobile agent integrity without any introduction of TTPs. Its design allows to achieve better performance and scalability than TTP solutions. Both protocols presents drawbacks, and we also present a combined approach that can overcome these limits. The protocols have been integrated in SOMA, a Secure and Open Mobile Agent environment that favours the design and the implementation of secure applications for open and global systems, such as the Internet and the Web.

\section{Acknowledgements}

Work carried out under the financial support of the Italian MURST in the framework of the Project "MOSAICO".

\section{References}

[1] Corradi, A., Cremonini, M. and Stefanelli, C., Security Models and Abstractions in a Mobile Agent Environment, Proceedings of IEEE WETICE98 Workshop on Collaboration in Presence of Mobility, USA, 1998.

[2] Corradi, A., Cremonini, M. and Stefanelli, C., Melding Abstractions with Mobile Agents, Proceedings of the $2^{\text {nd }}$ workshop Cooperative Information Agents (CIA98), LNAI 1435, Springer-Verlag, 1998.

[3] Freier, A., KARLton, P. and Kocher, P., SSL 3.0 Specification, http://home.netscape.com/eng/ssl3/index.html, 1996.

[4] Fuggetta, A., Picco, G.P. and Vigna, G., Understanding Code Mobility, IEEE Transaction on Software Engineering, Vol. 24, N. 5, 1998.

[5] Gong, L., Java Security: Present and Near Future, IEEE Micro, Vol.17, 1997.

[6] IAIK-JCE V2.0, Graz University, http://jcewww.iaik.tu-graz.ac.at/IAIK_JCE/

[7] Java development kit, Version 1.2 (beta 2), Sun Microsystems, 1998. http://java.sun.com/products/index.html. 
[8] Karjoth, G., Asokan, N., Gulcu, C., Protecting the Computation Results of FreeRoaming Agents, $2^{\text {nd }}$ International Workshop on Mobile Agents, 1998.

[9] Low, D., Protecting Java Code via Code Obfuscation, http://www.acm.org:81/crossroads/xrds4-3/codeob.html.

[10] MENEZES, A., et al., Handbook of Applied Cryptography, CRC Press, 1996.

[11] NeCulA, G., Proof Carrying code, Proceedings of the Twenty Fourth Annual Symposium on Principle of Programming Languages, 1997.

[12] Rотн, V., Secure Recording of Itineraries through Co-operative Agents, $4^{\text {th }}$ Workshop on Mobile Object Systems, 1998.

[13] Vigna, G. (Ed.), Mobile Agents and Security, LNCS 1419, Springer-Verlag, 1998.

[14] Wilhelm, U., Staamann, S., Buttyan, L., On the Problem of Trust in Mobile Agent Systems, Proceedings of NDSS'98, March 1998.

[15] YEE, B., A Sanctuary for Mobile Agents, Proceedings of the DARPA Workshop on Foundations for Secure Mobile Code, 1997.

\section{Biographies}

Antonio Corradi is an associate professor of computer science at the University of Bologna. His scientific interests include distributed systems, object and agent systems, network management, and distributed and parallel architectures. He received his Laurea in electronic engineering from the University of Bologna and his MS in electrical engineering from Cornell University. He is member of the ACM, AICA (Italian Association for Computing), and IEEE.

Marco Cremonini is a $\mathrm{PhD}$ student of computer science at the University of Bologna. His scientific interests include distributed systems, agent systems, security, and coordination. He received his Laurea in electronic engineering from the University of Bologna. He is student member of the ACM and IEEE.

Rebecca Montanari is currently a PhD student within DEIS, the Department of Electronics, Computer Science and Systems of the University of Bologna. Her scientific interests include distributed systems, object and agent systems, and network security management. She received her Laurea in electronic engineering from the University of Bologna. She is a student member of IEEE.

Cesare Stefanelli received the B.A. degree in Electronic Engineering from the University of Bologna, in Italy, in 1992 and the Ph.D. degree in Computer Science in 1996. His research interests are in the area of distributed systems, massively parallel systems and programming environments for parallelism. Within these areas of interest, he has taken and takes part to several Italian and international projects. Currently, he is associate professor of Operating Systems at the Faculty of Engineering of the University of Ferrara. 